\title{
Plane-Based Calibration of Cameras With Zoom Variation
}

\author{
Chao Yu, Gaurav Sharma \\ Electrical and Computer Engineering Dept \\ University of Rochester
}

\begin{abstract}
Plane-Based calibration algorithms have been widely adopted for the purpose of camera calibration task. These algorithms have the advantage of robustness compared to self-calibration and flexibility compared to traditional algorithms which require a $3 \mathrm{D}$ calibration pattern. While the common assumption is that the intrinsic parameters during the calibration process are fixed, limited consideration has been given to the general case when some intrinsic parameters maybe varying, others maybe fixed or known. We first discuss these general cases for camera calibration. Using a counting argument we enumerate all cases where plane-based calibration may be utilized and list the number of images required for each of these cases.

Then we extend the plane-based framework to the problem of cameras with zoom variation, which is the most common case. The approach presented and may be extended to incorporate additional varying parameters described in the general framework. The algorithm is tested using both synthetically generated images and actual images captured using a digital camera. The results indicate that the method performs very well and inherits the advantage of robustness and flexibility from plane-based calibration algorithms.
\end{abstract}

Keywords: Camera Calibration, Plane-based calibration, zoom lens

\section{INTRODUCTION}

The calibration of camera geometry is an essential step in a number of computer vision applications. The geometric calibration is summarized in the form of the camera projection matrix that is determined by a combination of intrinsic camera parameters and extrinsic orientation parameters.

A variety of techniques can be used for the purpose of camera calibration. The techniques can be classified in two main classes: 1) methods requiring the metric information of a calibration target and 2) methods that do not require the metric information of a calibration target and rely on scene content for the calibration (self-Calibration). Methods employing a calibration target require that the scene capture include a suitably designed target with known characteristics. Thus the target based methods are more intrusive. However, they typically also offer improved robustness and better parameter estimates for the calibration. For target based camera calibration techniques, the complexity of the calibration target is often a concern and methods for which the targets may be readily created are preferred. Thus plane-based calibration methods are of considerable interest. ${ }^{1,2}$ In this paper, we address plane-based calibration of camera with intrinsic parameter variations. The work is motivated by the observation that while extrinsic parameters typically vary between multiple image captures, the intrinsic parameters especially focal length (zoom) could possibly vary between multiple image captures.

Using a counting argument, we illustrate why zoom variation(and possibly others) in a camera (along with extrinsic parameter variation) may be calibrated by using correspondences between multiple views of a planar target captured with other intrinsic parameters fixed. Based on the planar homography model, ${ }^{3}$ we present an algorithm for the calibration of cameras with zoom variation using 4 or more views of the target. The method allows free translation of the camera and is easy to setup since only $2 D$ planar pattern is needed. The method generalizes the camera calibration technique developed in literature ${ }^{1,2}$ to incorporate an additional focal length parameter corresponding to the zoom which varies across the different images captured.

Further author information: (Send correspondence to Chao Yu): E-mail: chyu@ece.rochester.edu, Telephone: 1585 275 8122, Address: Electrical and Computer Engineering Department, University of Rochester, Rochester, NY, 146270126, USA, WWW: www.ece.rochester.edu/projects/iplab. This work is supported by the National Science Foundation under grant number ECS-0428157.

Visual Communications and Image Processing 2006, edited by John G. Apostolopoulos, Amir Said,

Proc. of SPIE-IS\&T Electronic Imaging, SPIE Vol. 6077, 607710, @ 2005 SPIE-IS\&T · 0277-786X/05/\$15 
We test the algorithm on simulated images captured from a synthetic scene and on real world scenes captured using a handheld digital camera. The accuracy of calibration is compared against common calibration methods.

The proposed approach is distinct from those in literature. ${ }^{4-7}$ Some work attacked the problem in a selfcalibration way under the assumption that the camera moves in pure rotation, ${ }^{5,6}$ others may required a $3 D$ calibration pattern. ${ }^{4,7}$

The paper is organized as following: section II present the camera model used which is the widely accepted one. A counting argument is presented in section III, section IV details the algorithm to perform calibration for zooming cameras. Both synthetic and real experiments are described in section V. In section VI, we discuss some practical issues with zoom lens. Finally the paper is concluded in section VII.

\section{CAMERA MODEL}

In homogeneous vector representation, the image plane coordinate of a $3 D$ point with coordinates $M$ is given by $^{3}$ :

$$
\mathbf{m}=\mathbf{K}[\mathbf{R} \mid \mathbf{t}] M=P M,
$$

where $\mathbf{K}$ stands for intrinsic parameters of the camera, $\mathbf{R}$ is the rotation matrix of the camera with respect to the world coordinate, $\mathbf{t}$ is the translation vector, and $P=\mathbf{K}[\mathbf{R} \mid \mathbf{t}]$ is called the camera projection matrix. The intrinsic parameter matrix $\mathbf{K}$ consists of five parameters:

$$
\mathbf{K}=\left[\begin{array}{ccc}
\alpha f & c & u \\
0 & f & v \\
0 & 0 & 1
\end{array}\right]
$$

the aspect ratio $\alpha$, the skewing factor $c$, the principal point $u, v$ and the focal length $f$. Camera calibration is to estimate all the parameters in $\mathbf{K}$ and usually $\mathbf{R}$ and $\mathbf{t}$.

\section{COUNTING ARGUMENTS FOR PLANE-BASED CAMERA CALIBRATION}

Given a planar surface in the scene, different views of the scene are related to the 3-D world coordinates by means of the corresponding camera projection matrix which is defined up to a scale factor (due to the use of homogeneous representation), ${ }^{3}$ we refer to this relation as a homography. Using a model plane with a pattern, the corresponding homography may be estimated provided the pattern consists of at least 4 points whose relative locations are known up to a scale factor. Based on the estimated projection matrix, 8 Degree Of Freedoms can therefore be fixed ( 3 by 3 matrix, with one degree of freedom due to scaling factor). If all the intrinsic parameters are unknown and fixed. After exclusion of 6 extrinsic parameters( 3 for rotation and 3 for translation), we get two constraints on intrinsic parameters. Thus 3 planes will be enough to get the 5 intrinsic parameters. However, in general case, when intrinsic parameters are also varying, a general counting argument is needed. We consider the case that extrinsic parameters such as rotation and translation are free to change.

Now suppose that in addition to extrinsic camera parameters, the focal length of the camera is also allowed to vary cross captured images. This is a common scenario when the zoom is changed. We assume that focal length is changing without affecting other parameters at the beginning, further consideration will be given to the possible variation of principal points. ${ }^{4}$ Under this assumption, within each homography, after exclusion of 7 DOF (3 for rotation, 3 for translation, 1 for focal length) we can get one constraint on the unknown parameters. In this case, 4 homographs are needed to fully recover the 4 unknowns(skewing factor $c$, aspect ratio $\alpha$, principal point $u, v)$. The recovery of other parameters are relatively straightforward.

When some parameters are known, they can easily be incorporated so that less planes are needed. For example, When 3 intrinsic parameters are known $($ say $u, v, \alpha)$ while the other two are fixed, we only have two parameters to estimate, one plane will be enough to perform this task, this is similar with part of Tsai's work, ${ }^{8}$ in which the camera is partly calibrated with one planar target(principal point, aspect ratio are not estimated). In case some parameters are varying during the calibration process, new unknowns must be introduced, consequently more planes are needed to finish the calibration task. A straight forward argument is that when two parameters are changing, all the other parameters must be known to calibrate, since the 8 DOFs given by homography will 
Table 1. Examples of number of images needed for plane-based calibration under no restriction on extrinsic parameters

\begin{tabular}{c|c|c|c}
\hline Varying & Known & Fixed & Distinct Planes required \\
\hline$f$ & & $u, v, c, \alpha$ & 4 \\
& & $f, u, v, c, \alpha$ & 3 \\
$f$ & $c$ & $u, v, \alpha$ & 3 \\
$f$ & $c, \alpha$ & $u, v$ & 2 \\
& $c, \alpha$ & $f, u, v$ & 2 \\
& $f, u, v$ & $c, \alpha$ & 1 \\
& $u, v, c, \alpha$ & $f$ & 1 \\
\hline
\end{tabular}

be fully occupied by these unknown parameters(6 extrinsic and 2 varying intrinsic). If more than two intrinsic parameters are varying, plane-based algorithm will fail.

Using a generalized version of these arguments, an analytical expression for the number of planes needed for calibration can be determined as follows. Let $n$ stands for number of planes needed, $x$ stands for number of varying parameters, $y$ for number of known parameters, then the following equation need to be met:

$$
\left\{\begin{array}{l}
(2-x) \times n \geq(5-x-y) \\
0 \leq x \leq 2 \\
x \leq x+y \leq 5
\end{array}\right.
$$

Typical cases are summarized in Table 1. Note that the table gives a theoretical counting argument. However, it maybe slightly modified in practice in order to simplify computation (for instance to ensure linear methods). We will see this in next section when we apply the conclusion to zooming cameras. For self-calibration algorithms, a similar counting argument for the estimation of varying intrinsic parameters can be found in Pollefeys et al.. ${ }^{9}$

Our consideration is given to the scenario that camera is free to move during the calibration, if we restrict the movement of camera to pure rotation, ${ }^{5,6}$ more freedom will be allowed for intrinsic parameters.

\section{CALIBRATION OF CAMERAS WITH ZOOM VARIATION}

In this section, we consider the case that focal length of camera is varying during the capture of calibration images, the approach can be easily extended to more general case. Our development closely follows previous presentations on plane-based methods ${ }^{1,2}$ while incorporating appropriate generalization for the varying focal length. We get a initial guess by utilizing constraints from homographies, then the result is refined by nonlinear optimization.

\subsection{Initial Estimate}

In plane-based algorithm, we assume the images are on the $\mathbf{Z}=0$ plane to obtain

$$
s \mathbf{m}=\mathbf{H} M
$$

where $s$ denotes unknown scaling factor, set $\mathbf{m}=\left[\begin{array}{lll}u & v & 1\end{array}\right]^{T}$ stands for a $2 D$ point in the image plane, $M=\left[\begin{array}{llll}X & Y & 0 & 1\end{array}\right]^{T}$ stands for a $3 D$ point at $\mathbf{Z}=0$ in the world. By expanding the homography,we have:

$$
s\left[\begin{array}{l}
u \\
v \\
1
\end{array}\right]=\mathbf{K}\left[\begin{array}{llll}
\mathbf{r}_{1} & \mathbf{r}_{2} & \mathbf{r}_{3} & \mathbf{t}
\end{array}\right]\left[\begin{array}{c}
X \\
Y \\
0 \\
1
\end{array}\right]=\mathbf{K}\left[\begin{array}{lll}
\mathbf{r}_{1} & \mathbf{r}_{2} & \mathbf{t}
\end{array}\right]\left[\begin{array}{c}
X \\
Y \\
1
\end{array}\right]
$$

It follows that:

$$
\mathbf{H}=\lambda \mathbf{K}\left[\begin{array}{lll}
\mathbf{r}_{1} & \mathbf{r}_{2} & \mathbf{t}
\end{array}\right]
$$


Since the homography can be estimated up to a scaling factor $\lambda$,

$$
\left[\begin{array}{lll}
\mathbf{h}_{1} & \mathbf{h}_{2} & \mathbf{h}_{3}
\end{array}\right]=\lambda \mathbf{K}\left[\begin{array}{lll}
\mathbf{r}_{1} & \mathbf{r}_{2} & \mathbf{t}
\end{array}\right]
$$

then, from the ortho-normality of rotation matrix, we get two constraints on $\mathbf{K}$ :

$$
\begin{gathered}
\mathbf{h}_{1}^{T} \mathbf{K}^{-T} \mathbf{K}^{-1} \mathbf{h}_{2}=0 \\
\mathbf{h}_{1}^{T} \mathbf{K}^{-T} \mathbf{K}^{-1} \mathbf{h}_{1}=\mathbf{h}_{2}^{T} \mathbf{K}^{-T} \mathbf{K}^{-1} \mathbf{h}_{2}
\end{gathered}
$$

Let $w=\mathbf{K}^{-T} \mathbf{K}^{-1}$, then $w$ is a symmetric matrix which also has a geometric meaning of absolute conic. ${ }^{3}$ To facilitate the extraction of linear constraints, we ignored skew factor $c$ at beginning, the nonlinear optimization process can recover it with the assumption that skewing factor is quite small,which is practical in most cases. Note that the algorithm can deal with non-zero $c$, but computation will be more complicated. As mentioned before, if we made some simplifications, the practical computation maybe slightly different with theoretical counting argument. In our case, we need at least 3 planes to perform calibration task.

$$
w_{i}=\left[\begin{array}{ccc}
1 & 0 & -u \\
0 & \alpha^{2} & -\alpha^{2} v \\
-u & -\alpha^{2} v & \alpha^{2} f_{i}^{2}+\left(u^{2}+\alpha^{2} v^{2}\right)
\end{array}\right] \stackrel{\text { def }}{=}\left[\begin{array}{ccc}
1 & 0 & x_{1} \\
0 & x_{2} & x_{3} \\
x_{1} & x_{3} & x_{4}
\end{array}\right]
$$

define,

$$
\mathbf{x}=\left[1, x_{1}, x_{2}, x_{3}, x_{4}\right]^{T}
$$

First, we set:

$$
\mathbf{h}_{i}^{T} w \mathbf{h}_{j}=\mathbf{a}_{i j}^{T} \mathbf{x}
$$

with

$$
\mathbf{a}_{i j}=\left[h_{i 1} h_{j 1}, h_{i 3} h_{j 1}+h_{i 1} h_{j 3}, h_{i 2} h_{j 2}, h_{i 3} h_{j 2}+h_{i 2} h_{j 3}, h_{i 3} h_{j 3}\right]^{T} .
$$

Then Equation. 2 can be written as :

$$
\left[\begin{array}{c}
\mathbf{a}_{12}^{T} \\
\left(\mathbf{a}_{11}-\mathbf{a}_{22}\right)^{T}
\end{array}\right] \mathbf{x}=\mathbf{0}
$$

Since the focal length is varying, $x_{4}$ differs for each plane, we need to extract the relationship between $x_{1}, x_{2}, x_{3}$, set $\mathbf{x}^{\prime}=\left[1, x_{1}, x_{2}, x_{3}\right]^{T}$ :

set $\mathbf{a}_{11}-\mathbf{a}_{22}=\mathbf{b}_{12}$, then set:

$$
\left[\begin{array}{c}
\mathbf{a}_{12}(1) \mathbf{b}_{12}(5)-\mathbf{b}_{12}(1) \mathbf{a}_{12}(5) \\
\mathbf{a}_{12}(2) \mathbf{b}_{12}(5)-\mathbf{b}_{12}(2) \mathbf{a}_{12}(5) \\
\mathbf{a}_{12}(3) \mathbf{b}_{12}(5)-\mathbf{b}_{12}(3) \mathbf{a}_{12}(5) \\
\mathbf{a}_{12}(4) \mathbf{b}_{12}(5)-\mathbf{b}_{12}(4) \mathbf{a}_{12}(5)
\end{array}\right]^{T} \mathbf{x}^{\prime}=0
$$

We can extract one linear equation between $x_{1}, x_{2}, x_{3}$. A geometric explanation is given in earlier work. ${ }^{10}$ The constraints between $u, v, c$ will be the center line. When several images are available, all the equations can be stacked together to form a linear equation.

$$
\mathbf{A x}^{\prime}=\mathbf{0}
$$

Solve this equation, we can get the initial guess of $u, v, \alpha$. In other words, all the center lines will intersect at one point which stands for the value of $u, v, \alpha$. Due to noise or other possible reason, such as the inaccuracy of camera model, accurate solution may not exist, a practical solution is to get the eigenvector of $\mathbf{A}$ associated with minimum eigenvalue. Other parameters can be easily recovered from the definition in Equation. 3. Then $\mathbf{r}_{1}=\frac{\mathbf{K}^{-1} \mathbf{h}_{1}}{\left\|\mathbf{K}^{-1} \mathbf{h}_{1}\right\|}, \mathbf{r}_{2}=\frac{\mathbf{K}^{-1} \mathbf{h}_{2}}{\left\|\mathbf{K}^{-1} \mathbf{h}_{2}\right\|}, \mathbf{r}_{3}=\mathbf{r}_{1} \times \mathbf{r}_{2}, \mathbf{t}=\frac{\mathbf{K}^{-1} \mathbf{h}_{3}}{\left\|\mathbf{K}^{-1} \mathbf{h}_{1}\right\|}$. 

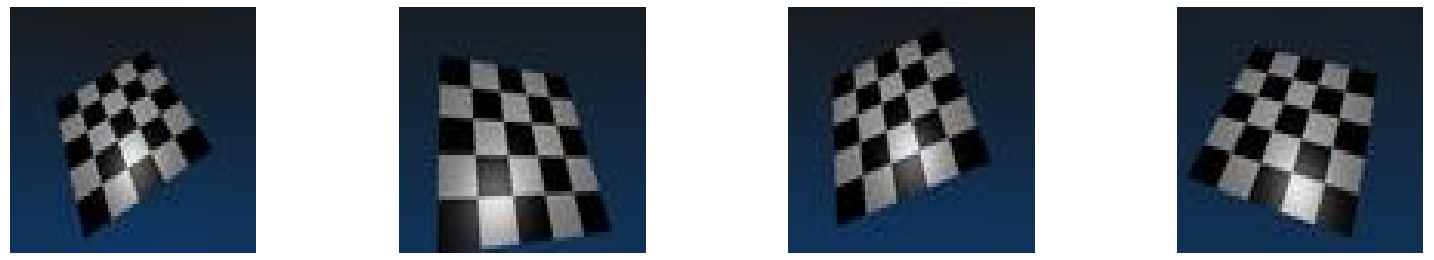

Figure 1. Images captured with synthetic data in Blender

\subsection{Refinement by Nonlinear Optimization}

After obtaining the initial estimate, we refine the estimate by nonlinear optimization procedure that additionally incorporate both the skewing factor $c$ (that was ignored at beginning) and radial distortion that may be present in captured images. As mentioned above, the skewing factor $c=0$ is a good initial estimate. When lens distortion is small, we can estimate other parameters as if the camera is distortion-free. nonlinear optimization could recover coefficients of distortion to a satisfactory level ${ }^{1}$ by considering the first two terms. The effect of radial lens distortion can be described as ${ }^{11}$ :

$$
\left\{\begin{array}{l}
\hat{x}=x+(x-u)\left(k_{1}\left(X_{c}^{2}+Y_{c}^{2}\right)+k_{2}\left(X_{c}^{2}+Y_{c}^{2}\right)^{2}\right) \\
\hat{y}=y+(y-v)\left(k_{1}\left(X_{c}^{2}+Y_{c}^{2}\right)+k_{2}\left(X_{c}^{2}+Y_{c}^{2}\right)^{2}\right)
\end{array}\right.
$$

Where $\hat{x}, \hat{y}$ stands for the real coordinate in image plane. $x, y$ stands for distortion free coordinate in image plane and $u, v$ are the principal points. Distortion coefficients are presented as $k_{1}, k_{2}$. Finally, $X_{c}, Y_{c}$ are the $2 \mathrm{D}$ coordinate in image plane before normalized to pixel value. We adopted Levenberg-Marquardt non-linear optimization algorithm $^{12}$ to refine the result which is available from the Minpack library. The cost function is defined as the Euclidean distance between back-projected and original points:

$$
\sum_{i=1}^{n} \sum_{j=1}^{m}\left\|m_{i j}-\hat{m}\left(\mathbf{K}, k_{1}, k_{2}, \mathbf{R}_{i}, \mathbf{t}_{i}, \mathbf{M}_{j}\right)\right\|^{2}
$$

In our implementation, homography is estimated by Direct Linear Transform(DLT). SVD decomposition is utilized to get a optimum solution to the linear equation. The initial value of distortion coefficients were set to be 0 . In Levenberg-Marquardt optimization, the $4+n$ (where $n$ stands for number of images, thus number of different focal lengths.) intrinsic parameters, 2 distortion coefficients, $6 n$ extrinsic parameters are optimized together. We also implemented the technique dealing with static focal length ${ }^{1}$ for comparison. In that case, $7+6 n$ parameters are optimized. The rotation matrix can be transformed into 3 independent parameters either by a Rodrigues way or from the definition of rotation matrix. ${ }^{8}$

\section{EXPERIMENTAL RESULTS}

\subsection{Synthetic Experiment}

In order to test the performance of the calibration method, we first performed simulations using the Blender modeling and rendering software package ${ }^{13}$ to capture synthetic images, ${ }^{14}$ which were then used for calibration. Four images of the target were captured with different zoom settings as in Figure. 1. The camera calibration parameters, including the different zoom settings, were estimated using the proposed algorithm. For the simulations, no radial distortion was assumed to be present. The results(without nonlinear optimization) are reported in Table 2 where the estimated values are also compared against the true values obtained from the settings within Blender. Note that in all cases the estimated values are close to the actual parameter settings, illustrating that the technique has the mathematical capability of recovering the true parameter values. 
Table 2. Camera calibration obtained using proposed method for simulated data obtained using Blender. Estimated and actual values are shown for four images captured with different zoom settings.

\begin{tabular}{|c|c|c|c|c|c|c|c|c|}
\hline \multirow{2}{*}{ Parameters } & \multicolumn{2}{|c|}{ Setting 1 } & \multicolumn{2}{c|}{ Setting 2 } & \multicolumn{2}{c|}{ Setting 3 } & \multicolumn{2}{c|}{ Setting 4 } \\
\cline { 2 - 8 } & Actual & Estimate & Actual & Estimate & Actual & Estimate & Actual & Estimate \\
\hline$f$ & 22.000 & 21.9748 & 30.000 & 29.9124 & 25.000 & 25.0109 & 35.000 & 34.8626 \\
\hline$\alpha$ & 1 & 1.0023 & 1 & 1.0023 & 1 & 1.0023 & 1 & 1.0023 \\
$u$ & 199.5 & 199.9176 & 199.5 & 199.9176 & 199.5 & 199.9176 & 199.5 & 199.9176 \\
$v$ & 149.5 & 147.9009 & 149.5 & 147.9009 & 149.5 & 147.9009 & 149.5 & 147.9009 \\
$c$ & 0 & 0 & 0 & 0 & 0 & 0 & 0 & 0 \\
\hline
\end{tabular}

Table 3. Experimental Results with Real Image

\begin{tabular}{|c|c|c|c|c|c|c|}
\hline & $f_{1}$ & $f_{2}$ & $f_{3}$ & $f_{4}$ & $u$ & $v$ \\
\hline Our Algorithm & 7201.0226 & 6800.7126 & 7985.7333 & 8118.2166 & 1470.6321 & 1124.4673 \\
\hline OpenCV Toolbox & 7091.5247 & 6752.1675 & 8057.5740 & 8057.5740 & $1508.8(42.3)$ & $1066.3(25.7)$ \\
\hline Relative Error(\%) & 1.55 & 0.71 & 0.89 & 0.76 & 2.6 & 5.2 \\
\hline
\end{tabular}

\subsection{Real Experiment}

We also conducted real experiments using a handheld digital camera. We compare the method to conventional plane-based calibration implemented in the camera calibration toolbox for matlab ${ }^{15}$ which is identical to the implementation used in the OpenCV library. ${ }^{16}$ Since the OpenCV method assumes a single focal length across multiple images, we captured multiple images with the same fixed focal length (no zoom variation). Estimates of camera calibration parameters were obtained with the proposed algorithm and with the OpenCV algorithm for comparison.

\subsubsection{Experiment with Captured Image}

Figure. 2 shows a set of images captured by an off-the-shelf Nikon D70. Experiment results are reported in Table. 3. Though the estimated parameters are reasonable, some accuracy is lost. A possible reason is that uncontrolled change and image processing within the camera may cause principal point $u, v$ to change along with the change in focal length $f$. For comparison of the techniques' accuracy, we avoid this problem by a simple method as indicated by next experiment. More detailed discussion can be found in the next section.
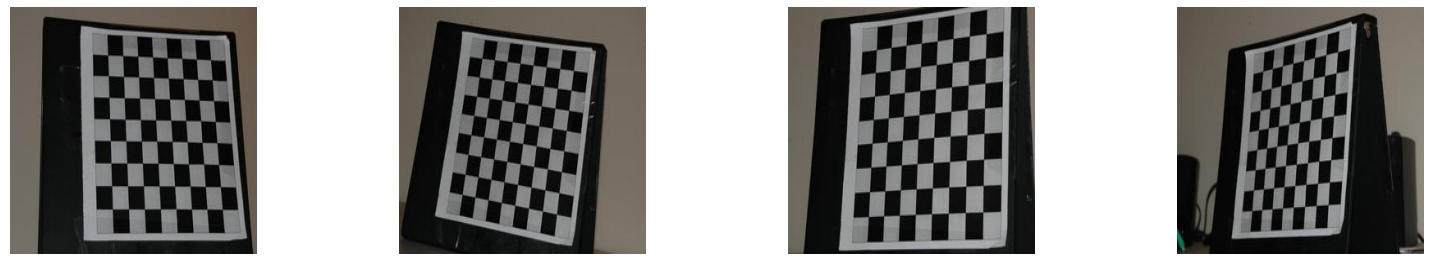

Figure 2. Images captured in experiments with real data.

\subsubsection{Experiment with multiple planes in one capture}

Several identical planar targets are created and captured simultaneously as illustrated in Figure. 3. $f, u, v$ are guaranteed to be fixed. A homography can be estimated from each plane. Other procedures of the calibration are exactly the same as presented in section.4. In other words, we created a $2.5 \mathrm{D}$ target to calibrate the camera. Four identical planes are utilized without information of exact layout. Note that the motivation is to calibrate zoom lens with possible variation of more than one intrinsic parameters during multiple captures. Table. 4 presents the experiment results. From the results, we can see that high accuracy is achieved.

\subsubsection{Experiment with Zhang's data}


Table 4. Experiment result with multiple planes captured simultaneously

\begin{tabular}{|c|c|c|c|c|c|c|c|c|}
\hline & $f_{1}$ & $f_{2}$ & $f_{3}$ & $f_{4}$ & $u$ & $v$ & $\alpha$ & $c$ \\
\hline Varying Focal Length: & 2878.4 & 2820.8 & 2907.9 & 2900.4 & 1066.7 & 844.2 & 0.9874 & 0.0286 \\
\hline Identical Focal Length: & \multicolumn{4}{|c|}{2855.5770} & 1047.9 & 844.8 & 0.9939 & 0.0156 \\
\hline
\end{tabular}

Table 5. Experimental Results with Zhang's Data ${ }^{1}$

\begin{tabular}{|c|c|c|c|c|c|c|c|}
\hline$f$ & $f_{1}$ & $f_{2}$ & $f_{3}$ & $f_{4}$ & $f_{5}$ & Mean & STD \\
\hline 831.81 & 849.8459 & 829.1820 & 830.5010 & 838.6833 & 838.2091 & 837.2843 & 8.2503 \\
\hline
\end{tabular}

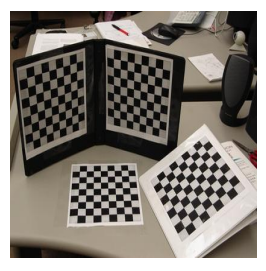

Figure 3. Multiple Planes

To facilitate comparison, we also conducted experiment with the data published by Zhang. ${ }^{1}$ The same data set is used to facilitate comparison. The results are reported in Table 5 and Table 6. Note that while the conventional method estimates only one focal length across the set of four images, the proposed algorithm estimates a focal length for each of the images (in addition to the other parameters). The results are quite close, which indicates that the method provides very good accuracy.

\section{DISCUSSION}

Our consideration is based on the assumption that only $f$ is changing. However, most of the amateur digital cameras are equipped with zoom lens. Calibrating these cameras could bring more challenges for the advanced features as auto-focusing, aperture adjusting, principal point shifting. When $f$ changes, other parameters probably may not remain static. Some early work ${ }^{7}$ has been done on this problem. According to the counting argument presented in section 3 , when $f, u, v$ are changing simultaneously, plane-based algorithm will fail, since 2 parameters are allowed to change with plane based method. We discuss several possibilities to deal with more varying parameters and other aspects of plane-based algorithms:

1. Vanishing points: vanishing points have been used for calibration in literature. ${ }^{17,18}$ However, it is easy to show that vanishing points can not give more constraints other than those we can extract from homography estimated. Similarly, metric information like known angle, known length ratio ${ }^{19}$ can not be used for more constraints.

2. Zoom lens Model: if a model for zoom lens exist, then the variation of $f, u, v$ will not be independent, knowledge about the model can be utilized as additional constraints.

3. Radial Alignment Constraint: Since radial distortion is also centered at the principal point. ${ }^{8,20}$ In the presence of radial distortion, the center of the image can be estimated by a non-linear optimization. ${ }^{20}$ Then we only need to estimate one parameter from homographies.

4. Calibration of multiple cameras: though variation of intrinsic parameters is a disadvantage in calibration, it can also bring new application. For multiple cameras in a camera network, when these cameras approximately share some intrinsic parameters, e.g. $\alpha, c, f$, they can be calibrated simultaneously with only one capture of planar target. On-line calibration is possible with assistance of a planar target without intervention with the $3 \mathrm{D}$ object.

5. Application in Image-based modeling: Shape from silhouette(SFS) has been of intensive interest recently. Prior work can be found in, ${ }^{21-24}$ in which a turn-table sequence or a 3D target is used for calibration. By using a planar object, the modeling processing will be more flexible.

Table 6. Experimental Results with Zhang's Data ${ }^{1}$ : Part 2

\begin{tabular}{|c|c|c|c|c|c|c|}
\hline & $\alpha$ & $c$ & $u$ & $v$ & $k_{1}$ & $k_{2}$ \\
\hline Zhang's result & 1.0000 & 0.2045 & 303.96 & 206.56 & -0.228 & 0.190 \\
\hline Our result & 0.9999 & 0.0005 & 303.9927 & 206.9484 & -0.2314 & 0.1983 \\
\hline
\end{tabular}




\section{CONCLUSIONS}

We consider the general framework for plane based camera calibration. Utilizing a counting argument we outline the different conditions under which plane based calibration may be utilized and also give an expression for the number of images required for the estimation of calibration parameters. An approach for calibration of cameras with zoom variations is detailed. To deal with the case that more than 2 parameters may be changing across multiple captures, a simple method by capturing multiple planes together without knowledge of layout is used to improve accuracy. Only planar calibration target is required, thus the algorithm is easy to setup. An initial guess of camera parameters are reached by solving linear equations, then nonlinear optimization is utilized to refine the result. Both synthetic and real experiment are carried out to show the effectiveness of the proposed algorithm.

\section{REFERENCES}

1. Z. Zhang, "Flexible camera calibration by viewing a plane from unknown orientations," in $I C C V$, pp. $666-$ $673,1999$.

2. P. F. Sturm and S. J. Maybank, "On plane-based camera calibration: A general algorithm, singularities, applications," in CVPR, pp. 432-437, 1999.

3. R. Hartley and A. Zisserman, Multiple view geometry in computer vision, Cambridge University Press, New York, NY, USA, 2000.

4. J.-M. Mengxiang Li, Lavest, "Some aspects of zoom lens camera calibration," Pattern Analysis and Machine Intelligence, IEEE Transactions on 18, pp. 1105-1110, Nov 1996.

5. R. S. Heung-Yeung Shum, "Panoramic image mosaics," Tech. Rep. MSR-TR-97, 1997.

6. L. Agapito, E. Hayman, and I. Reid, "Self-calibration of rotating and zooming cameras," Int. J. Comput. Vision 47(1-3), pp. 287-287, 2002.

7. R. G. Willson, Modeling and calibration of automated zoom lenses. PhD thesis, Pittsburgh, PA, USA, 1994.

8. R. Y. Tsai, "A versatile camera calibration technique for high-accuracy $3 \mathrm{~d}$ machine vision metrology using off-the-shelf tv cameras and lenses," Robotics and Automation, IEEE Journal of , pp. 323-344, 1987.

9. M. Pollefeys, R. Koch, and L. V. Gool, "Self-calibration and metric reconstruction inspite of varying and unknown intrinsic camera parameters," Int. J. Comput. Vision 32(1), pp. 7-25, 1999.

10. P. Gurdjos, A. Crouzil, and R. Payrissat, "Another way of looking at plane-based calibration: The centre circle constraint," in ECCV '02: Proceedings of the 7th European Conference on Computer Vision-Part IV, pp. 252-266, Springer-Verlag, (London, UK), 2002.

11. D. A. Forsyth and J. Ponce, Computer Vision: A Modern Approach, Prentice Hall, Englewood Cliffs, NJ, 2002.

12. J.More, "The levenberg-marquardt algorithm: Implementation and theory," Lecture Notes in Mathematics , 1977.

13. "Open source software package: Blender."

14. "Camera calibration for blender."

15. J. Bouguet, "Camera calibration toolbox for matlab."

16. "Open source computer vision library."

17. L.-L. Wang and W.-H. Tsai, "Camera calibration by vanishing lines for 3-d computer vision," IEEE Trans. Pattern Anal. Mach. Intell. 13(4), pp. 370-376, 1991.

18. B. Caprile and V. Torre, "Using vanishing points for camera calibration," Int. J. Comput. Vision 4(2), pp. 127-140, 1990.

19. D. Liebowitz and A. Zisserman, "Metric rectification for perspective images of planes," in CVPR '98: Proceedings of the IEEE Computer Society Conference on Computer Vision and Pattern Recognition, p. 482, IEEE Computer Society, (Washington, DC, USA), 1998.

20. R. K. Lenz and R. Y. Tsai, "Techniques for calibration of the scale factor and image center for high accuracy 3-d machine vision metrology," IEEE Trans. Pattern Anal. Mach. Intell. 10(5), pp. 713-720, 1988.

21. R. Szeliski, "Rapid octree construction from image sequences," CVGIP: Image Underst. 58(1), pp. 23-32, 1993. 
22. A. W. Fitzgibbon, G. Cross, and A. Zisserman, "Automatic 3d model construction for turn-table sequences," in SMILE'98: Proceedings of the European Workshop on 3D Structure from Multiple Images of Large-Scale Environments, pp. 155-170, Springer-Verlag, (London, UK), 1998.

23. S. J. Gortler, R. Grzeszczuk, R. Szeliski, and M. F. Cohen, "The lumigraph," in SIGGRAPH '96: Proceedings of the 23rd annual conference on Computer graphics and interactive techniques, pp. 43-54, ACM Press, (New York, NY, USA), 1996.

24. R. Wong, K.-Y.K. Cipolla, "Reconstruction of sculpture from its profiles with unknown camera positions," Image Processing, IEEE Transactions on 13(3), pp. 381-389, 2004. 